\title{
Excitonic enhancement of nonradiative energy transfer to bulk silicon with the hybridization of cascaded quantum dots
}

Aydan Yeltik, Burak Guzelturk, Pedro Ludwig Hernandez-Martinez, Shahab Akhavan, and Hilmi Volkan Demir

Citation: Appl. Phys. Lett. 103, 261103 (2013);

View online: https://doi.org/10.1063/1.4858384

View Table of Contents: http://aip.scitation.org/toc/apl/103/26

Published by the American Institute of Physics

\section{Articles you may be interested in}

Comparing direct charge injection and Forster energy transfer into quantum dots in hybrid organic/inorganic quantum dot light emitting devices

Journal of Applied Physics 112, 034501 (2012); 10.1063/1.4740234

Spectroscopic evidence for nonradiative energy transfer between colloidal CdSe/ZnS nanocrystals and functionalized silicon substrates

Applied Physics Letters 98, 161904 (2011); 10.1063/1.3579545

Interference-enhanced infrared-to-visible upconversion in solid-state thin films sensitized by colloidal nanocrystals

Applied Physics Letters 110, 211101 (2017); 10.1063/1.4984136

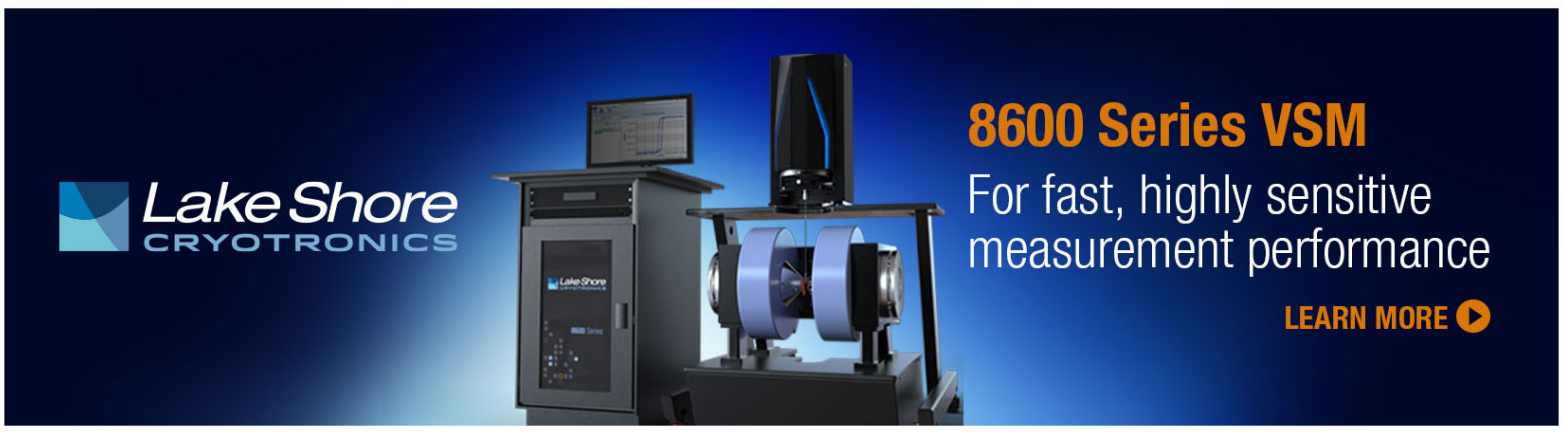




\title{
Excitonic enhancement of nonradiative energy transfer to bulk silicon with the hybridization of cascaded quantum dots
}

\author{
Aydan Yeltik, ${ }^{1}$ Burak Guzelturk, ${ }^{1}$ Pedro Ludwig Hernandez-Martinez,, ${ }^{1,2}$ Shahab Akhavan, ${ }^{1}$ \\ and Hilmi Volkan Demir ${ }^{1,2, a)}$ \\ ${ }^{1}$ Department of Physics and Department of Electrical and Electronics Engineering, UNAM-Institute \\ of Materials Science and Nanotechnology, Bilkent University, Ankara 06800, Turkey \\ ${ }^{2}$ Luminous! Center of Excellence for Semiconductor Lighting and Displays, School of Electrical \\ and Electronic Engineering and School of Physical and Mathematical Sciences, Nanyang Technological \\ University, Nanyang Avenue, Singapore 639798, Singapore
}

(Received 26 November 2013; accepted 5 December 2013; published online 23 December 2013)

\begin{abstract}
We report enhanced sensitization of silicon through nonradiative energy transfer (NRET) of the excitons in an energy-gradient structure composed of a cascaded bilayer of green- and red-emitting CdTe quantum dots (QDs) on bulk silicon. Here NRET dynamics were systematically investigated comparatively for the cascaded energy-gradient and mono-dispersed QD structures at room temperature. We show experimentally that NRET from the QD layer into silicon is enhanced by $40 \%$ in the case of an energy-gradient cascaded structure as compared to the mono-dispersed structures, which is in agreement with the theoretical analysis based on the excited state population-depopulation dynamics of the QDs. (C) 2013 AIP Publishing LLC. [http://dx.doi.org/10.1063/1.4858384]
\end{abstract}

Energy conversion efficiency is of great importance in light-harvesting systems including silicon ( $\mathrm{Si}$ ) based photovoltaic solar cells. ${ }^{1}$ For energy conversion, utilization of the optical spectrum by $\mathrm{Si}$ is limited due to being an indirect bandgap semiconductor. Previously, in order to enhance optical absorption of bulk $\mathrm{Si}$, several techniques have been proposed including light trapping, ${ }^{2}$ plasmonic field enhancement, ${ }^{3}$ and external light sensitization. ${ }^{4}$ For the efficiency enhancement, fluorescent sensitizers of $\mathrm{Si}$ using colloidal quantum dots (QDs) have been proposed owing to the tunable optical properties and large absorption cross-section of the QDs. Because of their superior properties, QDs are very promising materials for various optoelectronic applications. ${ }^{5-8}$ To this end, spectral sensitization of Si via QDs was demonstrated using both radiative and nonradiative energy transfer (NRET). NRET, which relies on the near-field dipole-dipole coupling, is a good candidate of directional energy flow in nanoscale systems. ${ }^{9-12}$

Previously, a monolayer of single type of QDs was employed to realize nonradiative sensitization of $\mathrm{Si}^{13}$ Furthermore, it has been recently shown that this type of NRET is phonon-assisted process unlike conventional NRET (or also known as Förster resonance energy transfer) systems of both fluorescent donor and acceptor. ${ }^{14}$ Since $\mathrm{Si}$ is an indirect bandgap material, phonon-assistance is required to realize nonradiative transfer of the excitation energy in the near-field. However, these types of QD-sensitized $\mathrm{Si}$ systems were always limited to a single type of QD donor. Alternatively, it has been reported that excitonic energy transfer among the QDs can be boosted using multilayer of QD structures with different sizes, which enables the energy gradient useful for exciton funneling. ${ }^{15-17}$ Using this exciton funneling, photoluminescence enhancement was achieved via inter-QD NRET in an energy-gradient structure. ${ }^{16}$ Such multi-layered QD solids were also used as an efficient platform for enhanced

\footnotetext{
${ }^{a)}$ E-mail: volkan@stanfordalumni.org. Telephone: +90 312 290-1021. Fax: +90 312 290-1123
}

exciton transfer from an epitaxial quantum well to the gradient-QD bilayer. ${ }^{18}$ Lee et al. demonstrated a cascadedNRET scheme to boost the absorption of CdTe nanowires via green and yellow CdTe QDs. ${ }^{19}$ A remarkable NRET from the outer-layer CdTe QDs into CdTe nanowires using chemical linkers was observed in their structures, resulting in a 4-fold luminescence enhancement of the nanowires. All these results indicate the great potential of these energy-channeling composite systems for the enhancement of exciton population in a luminescent acceptor.

In this study, different from the previous reports, we propose and demonstrate enhanced sensitization of silicon as a non-luminescent material technologically important for photovoltaics as well as photodetection without any need for using chemical linkers between QDs. To this end, we used the energy-gradient structure composed of a hybrid bilayered construct of green- and red-emitting CdTe QDs (GQDs and RQDs, respectively) on bulk Si. Dynamics of NRET from the QDs into $\mathrm{Si}\left(\mathrm{NRET}_{\mathrm{QD} \rightarrow \mathrm{Si}}\right.$ ) for such a cascaded structure with two types of QDs were investigated and compared to those for the structures with mono-dispersed QDs. Here, we find substantially enhanced $\mathrm{NRET}_{\mathrm{QD} \rightarrow \mathrm{Si}}$ using $\mathrm{Si}$ with the energy-gradient structure as compared to the mono-dispersed QD structures, which suggests the increased exciton population of the QDs closer to $\mathrm{Si}$ in the energy-gradient structure. Energy-gradient structure enables the migration of a favorably greater number of excitons from GQDs into RQDs to transfer into $\mathrm{Si}$, which leads to the improved sensitization in addition to enhanced overall NRET efficiency. Resultant significant enhancement of exciton population in Si offers an advantageous route for sensitization of Si via NRET, which was previously limited to mono-dispersed and single monolayer cases.

Herein, CdTe GQDs and RQDs were synthesized with the respective radii of 1.29 and $1.86 \mathrm{~nm}$, according to the procedure reported by Rogach et $a{ }^{20}{ }^{20}$ We fabricated hybrid nanostructures using layer-by-layer (LBL) deposition technique 
(a)

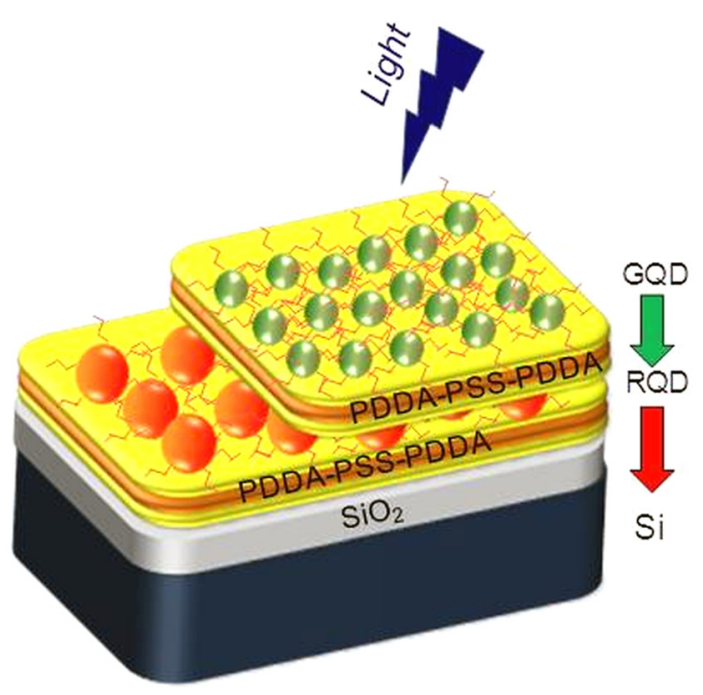

(b)

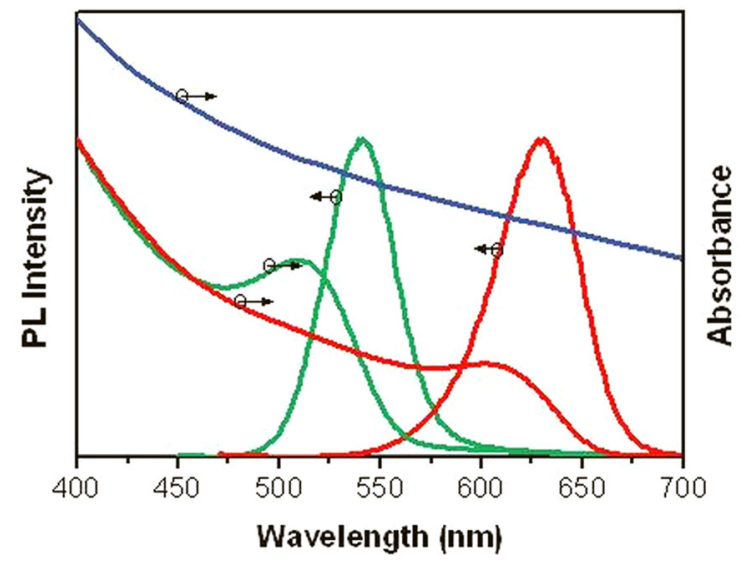

FIG. 1. (a) Schematic for the gradient energy transfer from the cascaded GQD/RQD bilayer into bulk Si. (b) Emission and absorption spectra of CdTe GQDs and CdTe RQDs (green and red curves, respectively), and absorption spectrum of bulk Si (blue curve).

(see supplementary material $^{21}$ ). After LbL-deposition, samples were kept for $3.5 \mathrm{~h}$ in a glovebox for complete drying and in dark to prevent photodegradation. Fig. 1(a) illustrates the generic architecture of the samples consisting of cascaded GQD and RQD bilayer separated by consecutive adsorption of poly(diallyldimethylammonium chloride) (PDDA) and poly (sodium 4-styrenesulfonate) (PSS) polyelectrolytes. Here the PDDA-PSS-PDDA stack has a thickness of $\sim 2.2 \mathrm{~nm}$ and suppresses charge transport. Si substrate possesses approximately $1.7 \mathrm{~nm}$ thick native oxide on the top, as verified by ellipsometry measurement. In addition, Fig. 1(b) depicts the emission and absorption spectra of GQDs and RQDs along with the absorption spectrum of bulk Si to illustrate the strong spectral overlap between the QDs and $\mathrm{Si}$, which is required to realize NRET. To assess the NRET among QDs $\left(\mathrm{NRET}_{\mathrm{GQD} \rightarrow \mathrm{RQD}}\right)$ and $\mathrm{NRET}_{\mathrm{QD} \rightarrow \mathrm{Si}}$, fluorescence decays of the QDs were recorded at room temperature by time resolved fluorescence (TRF) spectroscopy (PicoQuant FluoTime 200) with a pulsed laser at $375 \mathrm{~nm}$ as the excitation source. We prepared six different samples for a systematic study: (1) RQD/RQD/quartz, (2) GQD/GQD/quartz, (3) GQD/RQD/quartz, (4) RQD/RQD/Si, (5) GQD/GQD/Si, and (6) GQD/RQD/Si. For these structures, we investigated the emission dynamics of GQDs and RQDs recorded at their solid-state film peak emission wavelengths of 535 and $640 \mathrm{~nm}$, respectively. Due to the finite temporal response of the pulsed laser system, the decays were deconvoluted with the instrument response function. TRF decays were analyzed by $1 / \mathrm{e}$ fitting and lifetimes were obtained via amplitude-averaging of multi-exponentials $\left(\chi^{2} \sim 1\right)$. Samples (1), (2), and (3) are the references. However, quantum mechanically, a dipole nearby a different refractive index medium alters its radiative recombination dynamics. Therefore, the lifetimes are corrected in the case of quartz references considering the theory by Novotny and Hecht. ${ }^{22}$ In the lifetime analysis, $\mathrm{NRET}_{\mathrm{QD} \rightarrow \mathrm{Si}}$ rates were calculated using the expression $\gamma_{N R E T_{Q D-S i}}=\gamma_{\text {hybrid }}{ }_{Q D, S i}-\gamma_{\text {ref(corrected })_{Q D, Q \text { Quartz }}}$, where $\gamma_{\text {hybrid }}{ }_{Q D, S i}=1 / \tau_{\text {hybrid }_{Q D, S i}}$ is the excited state relaxation rate of either GQDs or RQDs in the hybrid structure and $\gamma_{\text {ref(corrected })}=1 / \tau_{\text {ref(corrected) } Q D, \text { Quartz }}$ is the recombination rate of the QDs in the quartz reference sample, where there is no NRET into the bulk-substrate. Efficiency of $\mathrm{NRET}_{\mathrm{QD} \rightarrow \mathrm{Si}}$ was found using the relation $\eta=\frac{\gamma_{N R E T}{ }_{Q D->S i}}{\gamma_{N R E T_{Q D->S i}}+\gamma_{\text {ref(corrected }) Q D, Q u a r z z}}$.

Exciton transfer dynamics of GQDs bilayer (GQD/GQD/Si) and cascaded bilayer on $\mathrm{Si}(\mathrm{GQD} / \mathrm{RQD} / \mathrm{Si})$, and their control groups on quartz (GQD/GQD/quartz and GQD/RQD/quartz) were investigated. As depicted in Fig. 2, we observed faster decays for the GQD/GQD/Si and GQD/RQD/Si samples as compared to their respective control groups, which is an indication of NRET from the QDs into Si. Moreover, $\mathrm{NRET}_{\mathrm{GQD} \rightarrow \mathrm{Si}}$ efficiency of $20 \%$ for the exciton migration from the GQDs into Si was calculated by using the lifetimes measured for the GQD/GQD/Si and GQD/GQD/quartz structures at $535 \mathrm{~nm}$. All the TRF lifetimes are presented in Table I. A considerable amount of exciton migration exists from GQD into RQD (with an NRET efficiency of $24 \%$ ) calculated by using the rates for the GQD/RQD/quartz and GQD/GQD/quartz structures. Furthermore, exciton population inside the GQDs decays faster for the GQD/RQD/Si sample as compared to that for the GQD/GQD/Si sample and this indicates an enhanced NRET as a result of the funneling effect in the cascaded structure.

In order to verify the gradient exciton feeding from the cascaded bilayered of differently sized QDs into Si, we further investigated the exciton transfer dynamics of the donor QDs at the RQD emission wavelength in detail. TRF decay curves of RQD/RQD/Si, GQD/RQD/Si and their respective

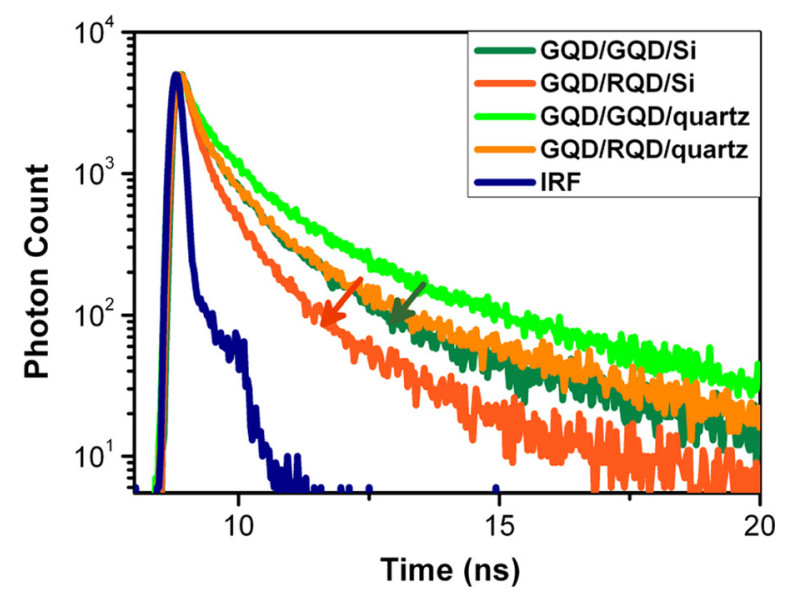

FIG. 2. TRF spectroscopy at $535 \mathrm{~nm}$ from the bilayer integration of GQDs on $\mathrm{Si}$ (dark green solid line), GQD/RQD on $\mathrm{Si}$ (dark orange solid line), GQDs on quartz (green solid line), GQD/RQD on quartz (orange solid line), and the laser diode response function (navy solid line). 
TABLE I. Fluorescence lifetimes for the QDs on quartz as the reference sample (corrected for refractive index variation) and on Si.

\begin{tabular}{cccc}
\hline \hline $\mathrm{GQD} / \mathrm{GQD} /$ quartz at $535 \mathrm{~nm}$ & $\mathrm{RQD} / \mathrm{RQD} /$ quartz at $640 \mathrm{~nm}$ & GQD/RQD/quartz at $535 \mathrm{~nm}$ & $\mathrm{GQD} / \mathrm{RQD} / \mathrm{quartz}$ at $640 \mathrm{~nm}$ \\
$0.444 \mathrm{~ns}$ & $1.763 \mathrm{~ns}$ & $0.338 \mathrm{~ns}$ & $2.262 \mathrm{~ns}$ \\
$\mathrm{GQD} / \mathrm{GQD} / \mathrm{Si}$ at $535 \mathrm{~nm}$ & $\mathrm{RQD} / \mathrm{RQD} / \mathrm{Si}$ at $640 \mathrm{~nm}$ & $\mathrm{GQD} / \mathrm{RQD} / \mathrm{Si}$ at $535 \mathrm{~nm}$ & $\mathrm{GQD} / \mathrm{RQD} / \mathrm{Si}$ at $640 \mathrm{~nm}$ \\
$0.363 \mathrm{~ns}$ & $0.350 \mathrm{~ns}$ & $0.263 \mathrm{~ns}$ & $0.707 \mathrm{~ns}$ \\
\hline \hline
\end{tabular}

control groups on quartz substrates are presented in Fig. 3. We obtained NRET $\mathrm{RQD}_{\mathrm{S}} \mathrm{Si}$ efficiency of $80 \%$ for the exciton migration from the RQDs into Si by using the lifetimes for the RQD/RQD/Si and RQD/RQD/quartz structures measured at $640 \mathrm{~nm}$ (Table I). In the context of gradient energy feeding, we observed an increased RQD lifetime for the cascaded GQD/RQD/Si structure as compared to that for the $\mathrm{RQD} / \mathrm{RQD} / \mathrm{Si}$ sample. This is owing to the strong exciton migration into RQDs via $\mathrm{NRET}_{\mathrm{GQD} \rightarrow \mathrm{RQD}}$, which is formed using the gradient energy feeding system resulting in higher exciton population in $\mathrm{Si}$.

To examine the experimentally observed NRET behaviors, relative exciton populations in $\mathrm{Si}$ were calculated using the efficiencies extracted from the TRF rates. The GQD lifetime is shortened down to $0.263 \mathrm{~ns}$ in the GQD/RQD/Si structure, while it is measured to be $0.444 \mathrm{~ns}$ in the GQD/GQD/quartz structure as the reference sample (Table I). By using these data, we estimate an exciton transfer rate of $\gamma_{N R E T_{G Q D->R Q D}}=1.550 \mathrm{~ns}^{-1}$ and the $\mathrm{NRET}_{\mathrm{GQD} \rightarrow \mathrm{RQD}}$ efficiency is calculated to be around $41 \%$. Based on this result and $\mathrm{NRET}_{\mathrm{RQD}} \rightarrow$ Si efficiency (80\%), we found the exciton populations for the GQD/RQD/Si structure (Case 1) and RQD/RQD/Si structure (Case 2). Comparing the population levels with each other, the enhancement factor (Eq. (9), supplementary material $\left.{ }^{21}\right)$ for the exciton transfer into Si ((\# of excitons for Case 1-\# of excitons for Case 2)/\# of excitons for Case 2) was found to be $\sim 40 \%$. Moreover, the enhancement factor is $\sim 450 \%$ when the exciton population for the GQD/RQD/Si structure is compared with the exciton population for the GQD/GQD/Si structure. This relatively huge factor probably stems from the efficient use of the trapped excitons in surface states of the GQDs, which have more trap states at the surface resulting from the synthesis process. ${ }^{15}$

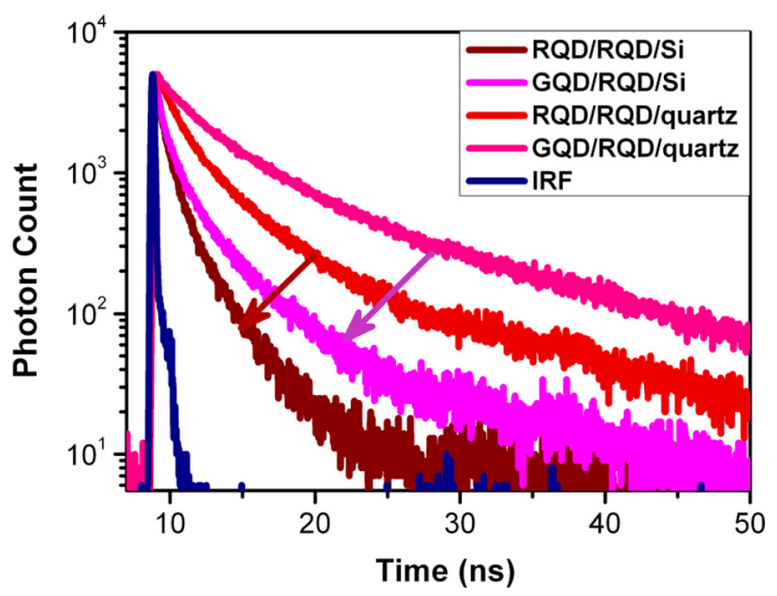

FIG. 3. TRF spectroscopy at $640 \mathrm{~nm}$ from bilayer integration of RQDs on $\mathrm{Si}$ (wine solid line), GQD/RQD on Si (magenta solid line), RQDs on quartz (red solid line), GQD/RQD on quartz (pink solid line), and the laser diode response function (navy solid line).
Both of these results ( $\sim 40 \%$ and $\sim 450 \%$ ) remain approximately the same when we calculate the population for the GQD/RQD/Si structure using $\mathrm{NRET}_{\mathrm{GQD} \rightarrow \mathrm{RQD}}$ efficiency of $24 \%$ and $\mathrm{NRET}_{\mathrm{RQD} \rightarrow \mathrm{Si}}$ efficiency of $80 \%$.

To further investigate the importance of energy gradient for $\mathrm{NRET}_{\mathrm{QD} \rightarrow \mathrm{Si}}$, we also analyzed the decay curves from the GQD/GQD/Si sample, which has a slower GQD exciton population decay than its cascaded structure (Fig. 2). In this configuration, the TRF lifetime is measured to be $0.363 \mathrm{~ns}$ (Table I). Weaker exciton transfer with a lower rate of $\gamma_{N R E T_{G Q D-S S}}$ $=0.503 \mathrm{~ns}^{-1}$ is calculated using the GQD/GQD/quartz structure as the reference, and a smaller $\mathrm{NRET}_{\mathrm{GQD} \rightarrow \mathrm{Si}}$ efficiency $(18 \%)$ is observed as compared to the energy gradient structure $\left(\mathrm{NRET}_{\mathrm{GQD} \rightarrow \mathrm{RQD}}=41 \%\right)$. In the case of employing the cascaded structure, it is therefore possible to boost this exciton funneling owing to the better utilization of the excitons in the QD layer via directed exciton transfer.

Furthermore, a theoretical study was performed to investigate the exciton populations in $\mathrm{Si}$ as a result of the $\mathrm{NRET}_{\mathrm{QD} \rightarrow \mathrm{Si}}$ process. For this purpose, we used a model based on density matrix formulation of near-field interactions. The master equations corresponding to the density matrices are derived as

$$
\begin{gathered}
\frac{\partial \rho^{A B}}{\partial t}=-\frac{i}{\hbar}\left[V_{A B}, \rho^{A B}\right]-\left(N_{\Gamma}^{A B} \rho^{A B}+\rho^{A B} N_{\Gamma}^{A B}\right) \\
\frac{\partial \rho^{B C}}{\partial t}=-\frac{i}{\hbar}\left[V_{B C}, \rho^{B C}\right]-\left(N_{\Gamma}^{B C} \rho^{B C}+\rho^{B C} N_{\Gamma}^{B C}\right)+P_{A B}\left[\rho^{A B}\right] \\
\frac{\partial \rho^{C}}{\partial t}=-\left(N_{\Gamma}^{C} \rho^{C}+\rho^{C} N_{\Gamma}^{C}\right)+P_{B C}\left[\rho^{B C}\right]
\end{gathered}
$$

where $\rho^{A B}, \rho^{B C}$, and $\rho^{C}$ represent the density matrices with the energy level $E_{1}, E_{2}$, and $E_{3}$, respectively (Fig. 4(b), inset). Herein, $V_{A B}=\hbar U_{Q D_{1} \rightarrow Q D_{2}}$ is the interaction between the levels with energy $\mathrm{E}_{1}$ of the top $\mathrm{QD}$ layer $\left(\mathrm{QD}_{1}\right)$ and the bottom QD layer $\left(\mathrm{QD}_{2}\right) ; V_{B C}=\hbar U_{Q D_{2} \rightarrow S i}$ is the interaction between levels with energy $\mathrm{E}_{2} ; N_{\Gamma}^{A B}, N_{\Gamma}^{B C}$, and $N_{\Gamma}^{C}$ are the diagonal matrices whose diagonal elements are given by $\gamma_{\alpha}$ and $\Gamma_{\alpha} ; P_{A B}\left[\rho^{A B}\right]$ represents the relaxation from the energy level $\mathrm{E}_{1}$ to $\mathrm{E}_{2}$; and $P_{B C}\left[\rho^{B C}\right]$ represents the relaxation from the energy level $E_{2}$ to $E_{3}$ (see supplementary material ${ }^{21}$ for more details). Excitonic relaxation rates for the GQDs and RQDs were obtained experimentally as $\gamma_{G Q D}=\frac{1}{0.444 \mathrm{~ns}}$ and $\gamma_{R Q D}=\frac{1}{1.763 n s}$, and, for $\mathrm{Si}$, it was taken as $\gamma_{S i}=\frac{1}{1 \mathrm{~ms}}$. The interaction rate between the QDs was taken as $U_{Q D_{1} \rightarrow Q D_{2}}=\frac{1}{100 n s} \cdot{ }^{18,23}$ The QD and Si relaxation rates were assumed to be $\Gamma_{Q D}=\frac{1}{20 p s}$ and $\Gamma_{S i}=\frac{1}{2 p s}$, which are reasonable values present in the literature. ${ }^{24,25}$ Finally, for the interaction rate between the QD and $\mathrm{Si}$, we used $U_{Q D_{2} \rightarrow S i}=\frac{1}{437 n s}$. Fig. 4(a) shows the exciton population (in \%) for the cases 
(a)

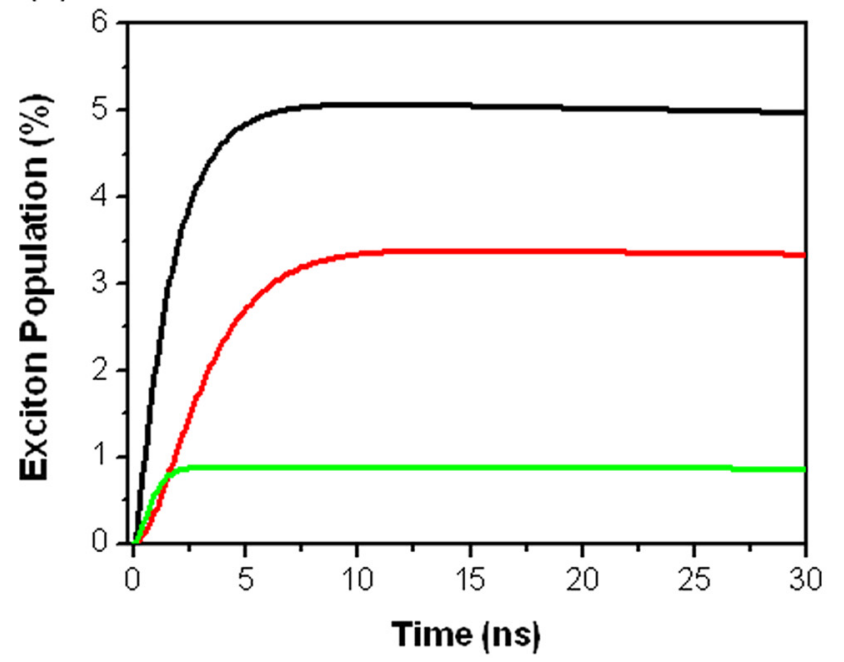

(b)

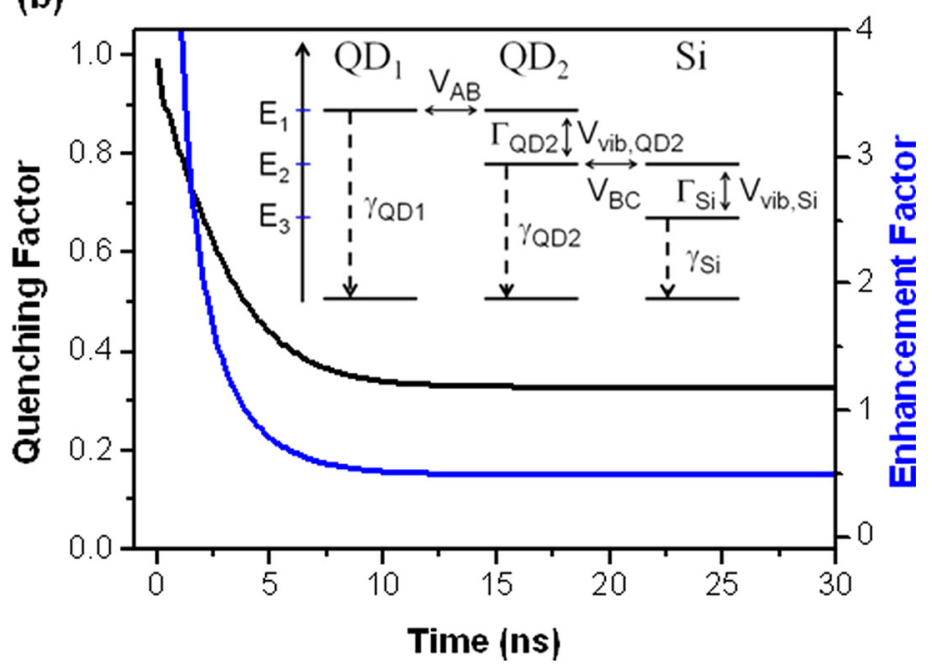

FIG. 4. (a) Exciton population for the cases of: (1) $G Q D \rightarrow R Q D \rightarrow S i$ (black solid line); (2) $R Q D \rightarrow R Q D \rightarrow S i$ (red solid line); and (3) $G Q D \rightarrow G Q D \rightarrow S i$ (green solid line). (b) Excitonic quenching factor (left) and enhancement factor (right) as a function of time for Case 1 with respect to Case 2.

of: (1) $G Q D \rightarrow R Q D \rightarrow S i$; (2) $R Q D \rightarrow R Q D \rightarrow S i$; and (3) $G Q D \rightarrow G Q D \rightarrow S i$. As it is shown, the best case for exciton transfer is given by Case 1, where the exciton transfer is cascaded from the GQD into the RQD and, at the end, into Si. The worst case corresponds to Case 3, where the exciton is transferred from the GQD in the first layer into the GQD in the second layer and, finally, into Si. The relative decrease in the exciton population for Case 1 with respect to Case 2 ((\# of excitons for Case 1-\# of excitons for Case 2)/\# of excitons for Case 1) is given in Fig. 4(b) as the quenching factor (Eq. (10), supplementary material ${ }^{21}$ ). The enhancement factor for Case 1 with respect to Case 2 is also depicted in Fig. 4(b). As given in this figure, the enhancement factor reaches up to $\sim 50 \%$ at the steady state condition, which is similar to the aforementioned experimental value of $40 \%$ (more details are given in the supplementary material ${ }^{21}$ ). All in all, the theoretical model is in good agreement with the experimental results and all these findings offer a strong evidence for the formation of gradient energy transfer, which leads to higher exciton population inside Si.

In summary, we demonstrated strong enhancement in the exciton population transferred into $\mathrm{Si}$ using the energy-gradient hybrid structures composed of cascaded bilayered green- and red-emitting QDs on Si. NRET dynamics were investigated for the cascaded structure and the structures with mono-dispersed QDs. For the cascaded architecture, exciton transfer efficiency of $41 \%$ from the GQDs into the RQDs and of $80 \%$ from the RQDs into Si was achieved at room temperature. As compared to the exciton population for the RQD/RQD/Si structure, the enhancement factor of exciton transfer into $\mathrm{Si}$ for the cascaded structure was found to be $\sim 1.4$ (40\%), which is in good agreement with the theoretical results of $\sim 1.5(50 \%)$. All these findings suggest that the energy-gradient structures with hybridized QDs on top of Si allow for high efficiency, which can be utilized in photovoltaic, photodetection, and possibly other optoelectronic hybrid devices.

\footnotetext{
${ }^{1}$ A. Müller, M. Ghosh, R. Sonnenschein, and P. Woditsch, Mater. Sci. Eng. B 134, 257 (2006).
}

${ }^{2}$ E. C. Garnett and P. Yang, J. Am. Chem. Soc. 130, 9224 (2008).

${ }^{3}$ H. A. Atwater and A. Polman, Nature Mater. 9, 205 (2010).

${ }^{4}$ B. Guzelturk, E. Mutlugun, X. Wang, K. L. Pey, and H. V. Demir, Appl. Phys. Lett. 97, 093111 (2010).

${ }^{5}$ S. V. Gaponenko, Optical Properties of Semiconductor Nanocrystals (Cambridge University Press, 1998).

${ }^{6}$ S. Akhavan, B. Guzelturk, V. K. Sharma, and H. V. Demir, Opt. Express 20, 25255 (2012).

${ }^{7}$ S. A. McDonald, G. Konstantatos, S. Zhang, P. W. Cyr, E. J. D. Klem, L. Levina, and E. H. Sargent, Nature Mater. 4, 138 (2005).

${ }^{8}$ U. Woggon, Optical Properties of Semiconductor Quantum Dots (Springer, 1997).

${ }^{9}$ T. Förster, Annu. Phys. 437, 55 (1948).

${ }^{10}$ M. Stavola, L. D. Dexter, and S. R. Knox, Phys. Rev. B 31, 2277 (1985).

${ }^{11}$ S. Lu and A. Madhukar, Nano Lett. 7, 3443 (2007).

${ }^{12}$ S. Lu, Z. Lingley, T. Asano, D. Harris, T. Barwicz, S. Guha, and A. Madhukar, Nano Lett. 9, 4548 (2009).

${ }^{13}$ H. M. Nguyen, O. Seitz, D. Aureau, A. Sra, N. Nijem, Y. N. Gartstein, Y. J. Chabal, and A. V. Malko, Appl. Phys. Lett. 98, 161904 (2011).

${ }^{14}$ A. Yeltik, B. Guzelturk, P. L. H. Martinez, A. Govorov, and H. V. Demir, "Phonon-assisted exciton transfer into silicon using nanoemitters: The role of phonons and temperature effects in förster resonance energy transfer," ACS Nano (published online).

${ }^{15}$ T. Franzl, T. A. Klar, S. Schietinger, A. L. Rogach, and J. Feldmann, Nano Lett. 4, 1599 (2004).

${ }^{16}$ T. A. Klar, T. Franzl, A. L. Rogach, and J. Feldmann, Adv. Mater. 17, 769 (2005).

${ }^{17}$ B. Guzelturk, P. L. H. Martinez, Q. Zhang, Q. Xiong, H. Sun, X. W. Sun, A. O. Govorov, and H. V. Demir, "Excitonics of semiconductor quantum dots and wires for lighting and displays," Laser Photonics Rev. (published online).

${ }^{18}$ S. Nizamoglu, P. Ludwig Hernández-Martínez, E. Mutlugun, D. Ugur Karatay, and H. Volkan Demir, Appl. Phys. Lett. 100, 241109 (2012).

${ }^{19}$ J. Lee, A. O. Govorov, and N. A. Kotov, Nano Lett. 5, 2063 (2005).

${ }^{20}$ A. L. Rogach, T. Franzl, T. A. Klar, J. Feldmann, N. Gaponik, V. Lesnyak, A. Shavel, A. Eychmüller, Y. P. Rakovich, and J. F. Donegan, J. Phys. Chem. C 111, 14628 (2007).

${ }^{21}$ See supplementary material at http://dx.doi.org/10.1063/1.4858384 for more details about the layer by layer deposition procedure and for more details about the theoretical analysis.

${ }^{22}$ L. Novotny and B. Hecht, Principles of Nano-Optics (Cambridge University Press, 2006).

${ }^{23}$ M. Naruse, E. Runge, K. Kobayashi, and M. Ohtsu, Phys. Rev. B 82, 125417 (2010).

${ }^{24}$ U. Bovensiepen, H. Petek, and M. Wolf, Dynamics at Solid State Surfaces and Interfaces: Volume 2: Fundamentals (Wiley-VCH, 2012).

${ }^{25}$ B. C. Daly, K. Kang, Y. Wang, and D. G. Cahill, Phys. Rev. B 80, 174112 (2009). 